\title{
Linearization coefficients of some particular Jacobi polynomials via hypergeometric functions
}

\section{Waleed M Abd-Elhameed ${ }^{*}$}

${ }^{*}$ Correspondence:

walee_9@yahoo.com Department of Mathematics, Faculty of Science, University of Jeddah, Jeddah, Saudi Arabia Department of Mathematics, Faculty of Science, Cairo University, Giza, Egypt

\section{照 Springer}

\begin{abstract}
The main purpose of the present paper is to establish two new linearization formulas for certain Jacobi polynomials. The new established formulas are expressed in terms of terminating hypergeometric functions of the type ${ }_{4} F_{3}(1)$. In virtue of the well-known Pfaff-Saalschütz identity, or by using some computer algebra algorithms, and in particular, the algorithms of Zeilberger, Petkovsek and van Hoeij, the resulting ${ }_{4} F_{3}(1)$ can be reduced for particular choices of the involved parameters. This reduction leads to obtaining several simple linearization formulas of some particular Jacobi polynomials free of any hypergeometric functions.
\end{abstract}

MSC: 33F10; 33C20; 33Cxx; 68W30

Keywords: Jacobi polynomials; linearization coefficients; generalized hypergeometric functions; computer algebra algorithms

\section{Introduction}

Linearization problems of orthogonal polynomials in general and of Jacobi polynomials in particular are of fundamental importance. Moreover, the connection problems between various orthogonal polynomials are also of old and recent interests. The linearization and connection coefficients problems of Jacobi polynomials have been extensively investigated by a large number of authors. For some studies in this direction, one can be referred to [1-8]. Some other studies about the connection and linearization problems for various orthogonal polynomials can be found in [9-14].

The class of Jacobi polynomials contains six important subclasses. The four polynomials, namely, the Chebyshev polynomials of the first and second kinds, the Legendre and ultraspherical polynomials are symmetric Jacobi polynomials, while the two polynomials, namely, the Chebyshev polynomials of third and fourth kinds are nonsymmetric Jacobi polynomials. The six special families of Jacobi polynomials are extensively used for numerically solving various kinds of boundary value problems (see, for example, [15-17]).

The linearization coefficients of the Jacobi polynomials are usually given in forms involving terminating hypergeometric functions. The Ph.D. dissertation of Tcheutia [18] exhibits a detailed study of the linearization coefficients of various orthogonal polynomials. Also, the author in his dissertation has discussed the connection and duplication problems of various classical orthogonal polynomials. Recently, Doha and Abd-Elhameed in [19] have

(c) $2016 \mathrm{Abd}$-Elhameed. This article is distributed under the terms of the Creative Commons Attribution 4.0 International License (http://creativecommons.org/licenses/by/4.0/), which permits unrestricted use, distribution, and reproduction in any medium, provided you give appropriate credit to the original author(s) and the source, provide a link to the Creative Commons license, and indicate if changes were made. 
derived in detail new linearization formulas of the third and fourth kinds of Chebyshev polynomials. They expressed these formulas in simple forms which are free of any hypergeometric functions, while Abd-Elhameed in [20, 21] and Abd-Elhameed et al. in [22] have developed some new linearization formulas for certain Jacobi polynomials.

Let $\left\{P_{n}\right\}_{n \geq 0}$ be a polynomial set and consider the two polynomials $A_{i}(x)$ and $B_{j}(x)$ with degrees $i$ and $j$, respectively, then the solution of the general linearization problem requires one to obtain the linearization coefficients $L_{i, j, k}$ such that

$$
A_{i}(x) B_{j}(x)=\sum_{k=0}^{i+j} L_{i, j, k} P_{k}(x) .
$$

The linearization problem (1) has the following two important particular problems:

(i) The standard linearization problem, which is called a Clebsch-Gordan-type problem. The solution of this problem requires one to find the coefficients $\gamma_{i, j, k}$ such that

$$
A_{i}(x) A_{j}(x)=\sum_{k=0}^{i+j} \gamma_{i j, k} A_{k}(x) .
$$

(ii) The connection problem. This problem requires one to find the connection coefficients $\xi_{i, k}$ in the equation

$$
A_{i}(x)=\sum_{k=0}^{i} \xi_{i, k} P_{k}(x) .
$$

We can summarize the objectives of this paper in the following two items:

- Establishing some new linearization formulas of Jacobi polynomials for special choices of their parameters.

- Establishing some new linearization formulas of the four kinds of Chebyshev polynomials.

As far as we know, the derived formulas are traceless in the literature.

The rest of the paper is as follows. First, preliminaries and transformation formulas between some hypergeometric functions are displayed in Section 2. In Section 3, we derive in detail the main linearization formula of particular Jacobi polynomials. Moreover, in this section, and based on using Pfaff-Saalschütz identity, some reduced linearization formulas are also obtained. Section 4 is devoted to utilizing some symbolic computation, and in particular, the celebrated algorithms of Zeilberger, Petkovsek, and van Hoeij, for the sake of obtaining some new linearization formulas of the four kinds of Chebyshev polynomials in reduced forms. In Section 5, we show the importance of the developed formulas by presenting two applications of them.

\section{Preliminaries and used formulas}

This section is dedicated to presenting an overview on some basic properties of the classical Jacobi polynomials which are useful in the sequel. Besides, we present transformation formulas between some kinds of hypergeometric functions. 


\subsection{Some relevant properties of the classical Jacobi polynomials}

The classical Jacobi polynomials associated with the real parameters $(\gamma>-1, \delta>-1)$ (see Olver et al. [23] and Andrews et al. [24]), are a sequence of polynomials $P_{i}^{(\gamma, \delta)}(x), x \in[-1,1]$ $(i=0,1,2, \ldots)$, each respectively of degree $i$. From now on, we use the normalized Jacobi polynomials which were used in Refs. [5, 22, 25]. We have

$$
R_{j}^{(\gamma, \delta)}(x)=\frac{P_{j}^{(\gamma, \delta)}(x)}{P_{j}^{(\gamma, \delta)}(1)}=\frac{j !}{(\gamma+1)_{j}} P_{j}^{(\gamma, \delta)}(x)={ }_{2} F_{1}\left(\begin{array}{c|c}
-j, j+\gamma+\delta+1 & \frac{1-x}{2} \\
\gamma+1 &
\end{array}\right) .
$$

The polynomials $R_{j}^{(\gamma, \delta)}(x)$ are orthogonal on $[-1,1]$ with respect to $w(x)=(1-x)^{\gamma}(1+x)^{\delta}$, in the sense that

$$
\begin{aligned}
& \int_{-1}^{1}(1-x)^{\gamma}(1+x)^{\delta} R_{j}^{(\gamma, \delta)}(x) R_{i}^{(\gamma, \delta)}(x) d x \\
& = \begin{cases}0, & j \neq i, \\
\frac{2^{\gamma+\delta+1} i ! \Gamma(i+\delta+1)[\Gamma(\gamma+1)]^{2}}{(2 i+\gamma+\delta+1) \Gamma(i+\gamma+\delta+1) \Gamma(i+\gamma+1)}, & j=i .\end{cases}
\end{aligned}
$$

It is worthy to mention here that the main advantage of using the normalized Jacobi polynomials which defined in (3) is that the six special polynomials of the Jacobi polynomials are given by the following simple forms:

$$
\begin{array}{lc}
C_{i}^{(\gamma)}(x)=R_{i}^{\left(\gamma-\frac{1}{2}, \gamma-\frac{1}{2}\right)}(x), & T_{i}(x)=R_{i}^{\left(-\frac{1}{2},-\frac{1}{2}\right)}(x), \\
U_{i}(x)=(i+1) R_{i}^{\left(\frac{1}{2}, \frac{1}{2}\right)}(x), & V_{i}(x)=R_{i}^{\left(-\frac{1}{2}, \frac{1}{2}\right)}(x), \\
W_{i}(x)=(2 i+1) R_{i}^{\left(\frac{1}{2},-\frac{1}{2}\right)}(x), & P_{i}(x)=R_{i}^{(0,0)}(x),
\end{array}
$$

where $C_{i}^{(\gamma)}(x), T_{i}(x), U_{i}(x), V_{i}(x), W_{i}(x)$, and $P_{i}(x)$ are the ultraspherical, Chebyshev of the first, second, third, and fourth kinds, and Legendre polynomials, respectively.

The following identity is also of interest:

$$
R_{i}^{(\gamma, \delta)}(-x)=\frac{(-1)^{i} \Gamma(\gamma+1) \Gamma(i+\delta+1)}{\Gamma(\delta+1) \Gamma(i+\gamma+1)} R_{i}^{(\gamma, \delta)}(x) .
$$

For more properties on Jacobi polynomials and their special polynomials, one can be referred to the important books [24] and [26].

\subsection{Some hypergeometric transformation formulas}

First, recall the well-known definition of the generalized hypergeometric function,

$$
\left.{ }_{p} F_{q}\left(\begin{array}{l}
{\left[a_{p}\right]} \\
{\left[b_{q}\right]}
\end{array}\right] \mid z\right)=\sum_{j=0}^{\infty} \frac{\left(a_{p}\right)_{j} z^{j}}{\left(b_{q}\right)_{j} j !}
$$

where the symbols $\left[a_{p}\right]$ and $\left[b_{q}\right]$ denote, respectively, the two sets $\left\{a_{1}, a_{2}, \ldots, a_{p}\right\}$ and $\left\{b_{1}, b_{2}, \ldots, b_{q}\right\}$ of complex or real parameters, where $b_{i} \neq 0$, for all $1 \leq i \leq q$, and $\left(a_{p}\right)_{k}=$ $\prod_{i=1}^{p}\left(a_{i}\right)_{k},\left(b_{q}\right)_{k}=\prod_{j=1}^{q}\left(b_{j}\right)_{k}$.

The following three theorems are of fundamental importance in establishing our results. 
Theorem 1 Let $m, p, q, r, s, t, u$ be nonnegative integers. The following transformation formula holds (see [27] and [28]):

$$
\begin{aligned}
{ }_{p+r+1} F_{q+s}\left(\begin{array}{c}
-m,\left[a_{p}\right],\left[c_{r}\right] \\
{\left[b_{q}\right],\left[d_{s}\right]}
\end{array} \mid z w\right)= & \sum_{j=0}^{m}\left(\begin{array}{c}
m \\
j
\end{array}\right) \frac{\left(a_{p}\right)_{j}\left(\alpha_{t}\right)_{j} z^{j}}{\left(b_{q}\right)_{j}\left(\beta_{u}\right)_{j}(j+v)_{j}} \\
& \times{ }_{p+t+1} F_{q+u+1}\left(\begin{array}{c}
j-m,\left[j+a_{p}\right],\left[j+\alpha_{t}\right] \\
2 j+v+1,\left[j+b_{q}\right],\left[j+\beta_{u}\right]
\end{array} \mid z\right) \\
& \times_{r+u+2} F_{s+t}\left(\begin{array}{c}
-j, j+v,\left[c_{r}\right],\left[\beta_{u}\right] \\
{\left[d_{s}\right],\left[\alpha_{t}\right]}
\end{array} \mid w\right) .
\end{aligned}
$$

Theorem 2 (Pfaff-Saalschütz identity (see [23])) For $c+d=a+b+1-m, m=0,1,2, \ldots$, one has

$$
{ }_{3} F_{2}\left(\begin{array}{c}
-m, a, b \\
c, d
\end{array} \mid 1\right)=\frac{(c-a)_{m}(c-b)_{m}}{(c)_{m}(c-a-b)_{m}} .
$$

Theorem 3 The following transformation formula holds (see [29]):

$$
{ }_{2} F_{1}\left(\begin{array}{c}
a, b \\
a+b-\frac{1}{2}
\end{array} \mid z\right){ }_{2} F_{1}\left(\begin{array}{c}
a-1, b \\
a+b-\frac{1}{2}
\end{array} \mid z\right)={ }_{3} F_{2}\left(\begin{array}{c}
2 a-1,2 b, a+b-1 \\
2 a+2 b-2, a+b-\frac{1}{2}
\end{array} \mid z\right) .
$$

\section{Establishing linearization formulas of particular Jacobi polynomials}

This section aims to establish new linearization formulas of products of some particular Jacobi polynomials.

Theorem 4 Let $n$ be a nonnegative integer. The following linearization formula is valid:

$$
\begin{aligned}
& R_{n}^{\left(\alpha, \frac{1}{2}\right)}(x) R_{n+1}^{\left(\alpha,-\frac{1}{2}\right)}(x) \\
& \quad=\sum_{j=0}^{2 n+1} \frac{\left(\begin{array}{c}
2 n+1 \\
j
\end{array}\right)\left(\alpha+\frac{1}{2}\right)_{j}(\gamma+1)_{j}(2 n+2 \alpha+3)_{j}}{(\alpha+1)_{j}(2 \alpha+1)_{j}(j+\gamma+\delta+1)_{j}} \\
& \quad \times{ }_{4} F_{3}\left(\begin{array}{c}
j-2 n-1, j+\alpha+\frac{1}{2}, j+2 n+2 \alpha+3, j+\gamma+1 \\
j+\alpha+1, j+2 \alpha+1,2 j+\gamma+\delta+2
\end{array} \mid 1\right) R_{j}^{(\gamma, \delta)}(x) .
\end{aligned}
$$

Proof First, the Gauss hypergeometric form of the orthogonal polynomials, $R_{n}^{(\alpha, \beta)}(x)$, enables one to write

$$
R_{n}^{\left(\alpha, \frac{1}{2}\right)}(x) R_{n+1}^{\left(\alpha,-\frac{1}{2}\right)}(x)={ }_{2} F_{1}\left(\begin{array}{c}
-n, n+\alpha+\frac{3}{2} \\
\alpha+1,
\end{array} \mid \frac{1-x}{2}\right){ }_{2} F_{1}\left(\begin{array}{c}
-n-1, n+\alpha+\frac{3}{2} \\
\alpha+1,
\end{array} \mid \frac{1-x}{2}\right),
$$

and in virtue of transformation (8), equation (10) can be turned into the form

$$
R_{n}^{\left(\alpha, \frac{1}{2}\right)}(x) R_{n+1}^{\left(\alpha,-\frac{1}{2}\right)}(x)={ }_{3} F_{2}\left(\begin{array}{c}
-2 n-1,2 n+2 \alpha+3, \alpha+\frac{1}{2} \\
2 \alpha+1, \alpha+1
\end{array} \mid \frac{1-x}{2}\right) .
$$


Now, if Theorem 1 is applied, accompanied with the choices

$$
\begin{aligned}
& p=q=2, \quad r=s=u=0, \quad t=1, \\
& {\left[c_{r}\right]=\left[d_{s}\right]=\left[\beta_{u}\right]=\emptyset, \quad z=1, \quad w=\frac{1-x}{2},}
\end{aligned}
$$

and if the remaining parameters in (6) are chosen suitably, then the following transformation formula is obtained:

$$
\begin{aligned}
{ }_{3} F_{2}\left(\begin{array}{c}
-2 n-1,2 n+2 \alpha+3, \alpha+\frac{1}{2} \\
2 \alpha+1, \alpha+1
\end{array} \mid \frac{1-x}{2}\right) \\
=\sum_{j=0}^{2 n} \frac{\left(\begin{array}{c}
2 n+1 \\
j
\end{array}\right)\left(\alpha+\frac{1}{2}\right)_{j}(\gamma+1)_{j}(2 n+2 \alpha+3)_{j}}{(\alpha+1)_{j}(2 \alpha+1)_{j}(j+\gamma+\delta+1)_{j}} \\
\quad \times{ }_{4} F_{3}\left(\begin{array}{c}
j-2 n-1, j+\alpha+\frac{1}{2}, j+2 n+2 \alpha+3, j+\gamma+1 \\
j+\alpha+1, j+2 \alpha+1,2 j+\gamma+\delta+2
\end{array} \mid 1\right) \\
\quad \times{ }_{2} F_{1}\left(\begin{array}{c}
-j, j+\gamma+\delta+1 \mid \frac{1-x}{2} \\
\gamma+1,
\end{array}\right),
\end{aligned}
$$

and this formula immediately yields (9).

Theorem 4 is now proved.

Now, we replace $x$ in (9) by $-x$, and with the aid of identity (5), the following linearization formula can easily be obtained.

Corollary 1 Let $n$ be a nonnegative integer. The following linearization formula is valid:

$$
\begin{aligned}
& R_{n}^{\left(\frac{1}{2}, \alpha\right)}(x) R_{n+1}^{\left(-\frac{1}{2}, \alpha\right)}(x) \\
& =\frac{(\alpha+1)_{n}(\alpha+1)_{n+1}}{\left(\frac{1}{2}\right)_{n+1}\left(\frac{3}{2}\right)_{n}} \sum_{j=0}^{2 n+1} \frac{(-1)^{j+1}\left(\begin{array}{c}
2 n+1 \\
j
\end{array}\right)\left(\alpha+\frac{1}{2}\right)_{j}(\delta+1)_{j}(2 n+2 \alpha+3)_{j}}{(\alpha+1)_{j}(2 \alpha+1)_{j}(j+\gamma+\delta+1)_{j}} \\
& \quad \times{ }_{4} F_{3}\left(\begin{array}{c}
j-2 n-1, j+\alpha+\frac{1}{2}, j+2 n+2 \alpha+3, j+\gamma+1 \\
j+\alpha+1, j+2 \alpha+1,2 j+\gamma+\delta+2
\end{array} \mid 1\right) R_{j}^{(\delta, \gamma)}(x) .
\end{aligned}
$$

Corollary 2 For the case corresponding to $\gamma=\alpha$ and $\delta=\frac{1}{2}$, the linearization formula (9) is reduced to the following formula:

$$
\begin{aligned}
& R_{n}^{\left(\alpha, \frac{1}{2}\right)}(x) R_{n+1}^{\left(\alpha,-\frac{1}{2}\right)}(x) \\
& =\sum_{j=0}^{2 n+1} \frac{\left(\begin{array}{c}
2 n+1 \\
j
\end{array}\right)(j+2)_{-j+2 n+1}\left(\alpha+\frac{1}{2}\right)_{j}\left(j-2 n-\alpha-\frac{1}{2}\right)_{-j+2 n+1}(2 n+2 \alpha+3)_{j}}{\left(j+\alpha+\frac{3}{2}\right)_{j}(2 \alpha+1)_{j}(-2 n-2 \alpha-1)_{-j+2 n+1}\left(2 j+\alpha+\frac{5}{2}\right)_{-j+2 n+1}} \\
& \quad \times R_{j}^{\left(\alpha, \frac{1}{2}\right)}(x) .
\end{aligned}
$$


Proof Substituting $\gamma=\alpha$ and $\delta=\frac{1}{2}$ into equation (9) yields

$$
\begin{aligned}
R_{n}^{\left(\alpha, \frac{1}{2}\right)}(x) R_{n+1}^{\left(-\frac{1}{2}, \alpha\right)}(x)= & \sum_{j=0}^{2 n+1} \frac{\left(\begin{array}{c}
2 n+1 \\
j
\end{array}\right)\left(\alpha+\frac{1}{2}\right)_{j}(2 n+2 \alpha+3)_{j}}{\left(j+\alpha+\frac{3}{2}\right)_{j}(2 \alpha+1)_{j}} \\
& \times{ }_{3} F_{2}\left(\begin{array}{c}
j-2 n-1, j+\alpha+\frac{1}{2}, j+2 \alpha+2 n+3 \\
2 j+\alpha+\frac{5}{2}, j+2 \alpha+1
\end{array} \mid 1\right) R_{j}^{\left(\alpha, \frac{1}{2}\right)}(x) .
\end{aligned}
$$

${ }_{3} F_{2}(1)$ in (15) is one-balanced, so it can be summed making use of Pfaff-Saalschütz identity (7) to give

$$
\begin{gathered}
{ }_{3} F_{2}\left(\begin{array}{c}
j-2 n-1, j+\alpha+\frac{1}{2}, j+2 \alpha+2 n+3 \mid 1 \\
2 j+\alpha+\frac{5}{2}, j+2 \alpha+1
\end{array}\right) \\
\quad=\frac{(j+2)_{-j+2 n+1}\left(j-2 n-\alpha-\frac{1}{2}\right)_{-j+2 n+1}}{(-2 n-2 \alpha-1)_{-j+2 n+1}\left(2 j+\alpha+\frac{5}{2}\right)_{-j+2 n+1}} .
\end{gathered}
$$

The last reduction formula enables one to write the linearization formula (15) in the reduced form given in (14).

Corollary 2 is proved.

The following two important linearization formulas can be obtained by setting, respectively, $\alpha=\frac{1}{2},-\frac{1}{2}$.

Corollary 3 Let $n$ be a nonnegative integer. The following two linearization formulas are valid:

$$
U_{n}(x) W_{n+1}(x)=\sum_{j=0}^{2 n+1} U_{j}(x)
$$

and

$$
V_{n}(x) T_{n+1}(x)=\frac{1}{2}\left(1+V_{2 n+1}(x)\right) .
$$

Remark 1 It is worthy to note here that the two linearization formulas (17) and (18) of Chebyshev polynomials can also be obtained from their trigonometric representations.

\section{Some new linearization formulas via employing computer algebra algorithms}

The main aim of this section is to establish some other new linearization formulas free of any hypergeometric functions. The basic idea upon deriving the desired linearization formulas is essentially based upon employing some symbolic computation such as Zeilberger's, Petkovsek's and van Hoeij's algorithms, for the sake of reducing the ${ }_{4} F_{3}(1)$, which appears in (9), for certain choices of the involved parameters. This leads to obtaining some new linearization formulas of Jacobi polynomials of particular parameters.

Corollary 4 Setting $\alpha=\frac{1}{2}$ and $\gamma=\delta=-\frac{1}{2}$ in (9) yields the following linearization formula:

$$
U_{n}(x) W_{n+1}(x)=2 \sum_{m=0}^{n} \frac{1}{\xi_{m}}(n+1-m) T_{2 m}(x)+2 \sum_{m=0}^{n}(n+1-m) T_{2 m+1}(x)
$$


where

$$
\xi_{m}= \begin{cases}2, & m=0 \\ 1, & m>0\end{cases}
$$

Proof Substituting $\alpha=\frac{1}{2}$ and $\gamma=\delta=-\frac{1}{2}$ into equation (9) yields

$$
\begin{aligned}
R_{n}^{\left(\frac{1}{2}, \frac{1}{2}\right)}(x) R_{n+1}^{\left(\frac{1}{2}, \frac{-1}{2}\right)}(x)= & \sum_{j=0}^{2 n+1} \frac{\left(\begin{array}{c}
2 n+1 \\
j
\end{array}\right)\left(\frac{1}{2}\right)_{j}(2 n+4)_{j}}{(j+1)\left(\frac{3}{2}\right)_{j}(j)_{j}} \\
& \times{ }_{4} F_{3}\left(\begin{array}{c}
j-2 n-1, j+\frac{1}{2}, j+1, j+2 n+4 \\
j+\frac{3}{2}, j+2,2 j+1
\end{array} \mid 1\right) T_{j}(x) .
\end{aligned}
$$

To the best of our knowledge, the ${ }_{4} F_{3}(1)$ in (21) cannot be summed by using any standard formulas in the literature, so we employ computer algebra for the desired reduction. If we set

$$
M_{\ell, n}={ }_{4} F_{3}\left(\begin{array}{c}
-\ell,-\ell+2 n+\frac{3}{2},-\ell+2 n+2,-\ell+4 n+5 \\
-\ell+2 n+\frac{5}{2},-\ell+2 n+3,-2 \ell+4 n+3
\end{array} \mid 1\right),
$$

then with the aid of Zeilberger's algorithm (see for instance, Koepf [30]), the third-order recurrence relation satisfied by $M_{\ell, n}$ with respect to $\ell$ is

$$
\begin{aligned}
& 64(\ell-2 n-2)_{3}\left(\ell-2 n-\frac{3}{2}\right)_{3} M_{\ell+3, n}+4(\ell+3)(\ell-4 n-2)(2 \ell-4 n-3)(2 \ell-4 n-1) \\
& \quad \times(\ell-2 n-2)_{2} M_{\ell+2, n}-2(\ell+2)_{2}(\ell-4 n-3)_{2}(2 \ell-4 n-3)(\ell-2 n-2) M_{\ell+1, n} \\
& \quad-(\ell+1)_{3}(\ell-4 n-4)_{3} M_{\ell, n}=0
\end{aligned}
$$

with the initial values

$$
M_{0, n}=1, \quad M_{1, n}=\frac{1}{4 n+3}, \quad M_{2, n}=\frac{2}{(2 n+1)(4 n+1)} .
$$

It can be shown that the third-order recurrence equation (22) has the following exact solution:

$$
M_{\ell, n}= \begin{cases}\frac{\ell !(\ell+2)(-2 \ell+4 n+4) !}{2(-\ell+4 n+4) !}, & \ell \text { even, } \\ \frac{(\ell+1) !(-2 \ell+4 n+4) !}{2 \xi_{2 n-\ell+1}(-\ell+4 n+4) !}, & \ell \text { odd },\end{cases}
$$

and therefore ${ }_{4} F_{3}(1)$ in (21) has the following reduction formula:

$$
{ }_{4} F_{3}\left(\begin{array}{cl}
j-2 n-1, j+\frac{1}{2}, j+1, j+2 n+4 \\
j+\frac{3}{2}, j+2,2 j+1
\end{array} \mid 1\right)= \begin{cases}\frac{(j+1)(2 j+1) !(-j+2 n+2) !}{\xi_{j}(j+2 n+3) !}, & j \text { even } \\
\frac{(-j+2 n+3)(2 j+2) !(-j+2 n+1) !}{2(j+2 n+3) !}, & j \text { odd }\end{cases}
$$

The last reduction formula along with equation (21)-after performing some manipulations-leads to the following linearization formula: 


$$
U_{n}(x) W_{n+1}(x)=2 \sum_{m=0}^{n} \frac{1}{\xi_{m}}(n+1-m) T_{2 m}(x)+2 \sum_{m=0}^{n}(n+1-m) T_{2 m+1}(x)
$$

Corollary 4 is proved.

Remark 2 It is worthy to note the 'sumrecursion command' in the Maple software may be employed to obtain the recurrence relation (22).

Remark 3 It is worthy to note here that the algorithm of Petkovsek (see Koepf [30], Chapter 9), or the improved version of van Hoeij [31], may be employed in order to obtain the exact solution (23) of the third-order recurrence equation (22).

Corollary 5 Setting $\alpha=\delta=\frac{1}{2}$ and $\gamma=-\frac{1}{2}$ in (9) yields the following linearization formula:

$$
U_{n}(x) W_{n+1}(x)=\sum_{j=0}^{2 n+1}(2 n+2-j) V_{j}(x)
$$

Proof Substituting $\alpha=\delta=\frac{1}{2}$ and $\gamma=-\frac{1}{2}$ into equation (9) yields

$$
\begin{aligned}
R_{n}^{\left(\frac{1}{2}, \frac{1}{2}\right)}(x) R_{n+1}^{\left(\frac{1}{2}, \frac{-1}{2}\right)}(x)= & \sum_{j=0}^{2 n+1} \frac{\left(\begin{array}{c}
2 n+1 \\
j
\end{array}\right)\left(\frac{1}{2}\right)_{j}(2 n+4)_{j}}{(j+1)\left(\frac{3}{2}\right)_{j}(j+1)_{j}} \\
& \times{ }_{4} F_{3}\left(\begin{array}{c}
j-2 n-1, j+\frac{1}{2}, j+1, j+2 n+4 \\
j+\frac{3}{2}, j+2,2 j+2
\end{array} \mid 1\right) V_{j}(x) .
\end{aligned}
$$

Now, if we set

$$
G_{\ell, n}={ }_{4} F_{3}\left(\begin{array}{c}
-\ell,-\ell+2 n+\frac{3}{2},-\ell+2 n+2,-\ell+4 n+5 \\
-\ell+2 n+\frac{5}{2},-\ell+2 n+3,-2 \ell+4 n+4
\end{array} \mid 1\right)
$$

then with the aid of Zeilberger's algorithm, the second-order recurrence relation satisfied by $G_{\ell, n}$ with respect to $\ell$ is

$$
\begin{aligned}
& (\ell+1)(\ell+2)(\ell-4 n-4)(\ell-4 n-3) G_{\ell, n} \\
& \quad+4(\ell+2)(\ell-4 n-3)(2 \ell-4 n-3)(\ell-2 n-2) G_{\ell+1, n} \\
& \quad+4(2 \ell-4 n-3)(2 \ell-4 n-1)(\ell-2 n-2)(\ell-2 n-1) G_{\ell+2, n}=0
\end{aligned}
$$

with the initial values

$$
G_{0, n}=1, \quad G_{1, n}=\frac{2}{4 n+3} .
$$

It can be shown that the recurrence equation (26) has the following exact solution:

$$
G_{\ell, n}=\frac{(\ell+1) !(-2 \ell+4 n+4) !}{(-\ell+4 n+4) !}
$$


and this accordingly implies that ${ }_{4} F_{3}(1)$ in $(25)$ has the following reduction formula:

$$
{ }_{4} F_{3}\left(\begin{array}{c}
j-2 n-1, j+\frac{1}{2}, j+1, j+2 n+4 \\
j+\frac{3}{2}, j+2,2 j+2
\end{array} \mid 1\right)=\frac{(2 j+2) !(2 n-j+2) !}{(2 n+j+3) !}
$$

The last reduction formula along with equation (25)-after performing some manipulations-lead to the following linearization formula:

$$
U_{n}(x) W_{n+1}(x)=\sum_{j=0}^{2 n+1}(2 n+2-j) V_{j}(x)
$$

Corollary 5 is proved.

Corollary 6 Setting $\alpha=-\frac{1}{2}$ and $\delta=\gamma$ in (9) yields the following linearization formula:

$$
\begin{aligned}
& V_{n}(x) T_{n+1}(x) \\
&= \frac{1}{2}+\frac{(-1)^{n+1} \Gamma(\gamma) \Gamma(\gamma+1)}{2 \Gamma(\gamma-n) \Gamma(n+\gamma+1)}+\frac{\sqrt{\pi} \Gamma(\gamma)}{2^{2 \gamma} \Gamma\left(\gamma+\frac{1}{2}\right)} \\
& \quad \times {\left[\sum_{m=0}^{n} \frac{(-1)^{m+n}(\gamma+2 m+1)(m+n+1) ! \Gamma(2 m+2 \gamma+1)}{(2 m+1) !(n-m) ! \Gamma(m-n+\gamma) \Gamma(m+n+\gamma+2)} C_{2 m+1}^{(\gamma)}\right.} \\
&\left.\quad+\sum_{m=1}^{n} \frac{(-1)^{m+n+1}(\gamma+2 m)(m+n) ! \Gamma(2 m+2 \gamma)}{(2 m) !(n-m) ! \Gamma(m-n+\gamma) \Gamma(m+n+\gamma+1)} C_{2 m}^{(\gamma)}(x)\right] .
\end{aligned}
$$

Proof At first, and with the aid of the Legendre duplication formula

$$
\frac{\Gamma(2 z)}{\Gamma(z)}=\frac{2^{2 z-1} \Gamma\left(z+\frac{1}{2}\right)}{\sqrt{\pi}}
$$

it is easy to see that

$$
\lim _{\alpha \rightarrow \frac{-1}{2}} \frac{\left(\alpha+\frac{1}{2}\right)_{j}}{(2 \alpha+1)_{j}}= \begin{cases}1, & j=0 \\ \frac{1}{2}, & j \geq 1\end{cases}
$$

and hence the linearization formula (9) for the case corresponds to the choice $\alpha=-\frac{1}{2}$ and $\delta=\gamma$ is turned into

$$
\begin{aligned}
V_{n}(x) T_{n+1}(x)= & \frac{1}{2}+\frac{1}{2} \sum_{j=0}^{2 n} \frac{\left(\begin{array}{c}
2 n+1 \\
j
\end{array}\right)(2 n+2)_{j}(\gamma+1)_{j}}{\left(\frac{1}{2}\right)_{j}(j+2 \gamma+1)_{j}} \\
& \times{ }_{3} F_{2}\left(\begin{array}{c}
j-2 n-1, j+2 n+2, j+\gamma+1 \\
j+\frac{1}{2}, 2 j+2 \gamma+2
\end{array} \mid 1\right) R_{j}^{(\gamma, \gamma)}(x) .
\end{aligned}
$$

Now, if we set

$$
M_{\ell, n, \gamma}={ }_{3} F_{2}\left(\begin{array}{c}
-\ell,-\ell+4 n+3, \gamma-\ell+2 n+2 \\
-\ell+2 n+\frac{3}{2}, 2 \gamma-2 \ell+4 n+4
\end{array} \mid 1\right)
$$


then in virtue of Zeilberger's algorithm, the second-order recurrence relation satisfied by $M_{\ell, n, \gamma}$ with respect to $\ell$ is

$$
\begin{aligned}
& (1-\ell)(2 \gamma-\ell+2)(\ell-4 n-4)(2 \gamma-\ell+4 n+5) M_{\ell-2, n, \gamma} \\
& \quad-(2 \ell-4 n-5)(2 \gamma-2 \ell+4 n+5)(2 \gamma-2 \ell+4 n+7) M_{\ell-1, n, \gamma} \\
& \quad+(2 \ell-4 n-5)(2 \ell-4 n-3)(2 \gamma-2 \ell+4 n+5)(2 \gamma-2 \ell+4 n+7) M_{\ell, n, \gamma}=0,
\end{aligned}
$$

with the initial values

$$
M_{0, n, \gamma}=1, \quad M_{1, n, \gamma}=\frac{-1}{1+4 n} .
$$

The exact solution of (30) is given by

$$
M_{\ell, n, \gamma}= \begin{cases}\frac{(-1)^{\frac{\ell}{2}} 2^{-\ell} \ell !\left(-\frac{\ell}{2}+\gamma+\frac{1}{2}\right) \frac{\ell}{2}}{\left(\frac{\ell}{2}\right) !\left(-\ell+2 n+\frac{3}{2}\right)_{\frac{\ell}{2}}\left(-\ell+2 n+\gamma+\frac{5}{2}\right)}, \frac{\ell}{2}, & \ell \text { even } \\ \frac{(-1)^{\frac{\ell+1}{2}} 2^{-\ell-1}(\ell+1) !\left(-\frac{\ell}{2}+\gamma+1\right) \frac{\ell-1}{2}}{\left(\frac{\ell+1}{2}\right) !\left(-\ell+2 n+\frac{3}{2}\right) \frac{\ell+1}{2}\left(-\ell+2 n+\gamma+\frac{5}{2}\right)}, \frac{\ell-1}{2}, & \ell \text { odd }\end{cases}
$$

and therefore the following linearization formula is obtained:

$$
V_{n}(x) T_{n+1}(x)=\frac{1}{2}+\frac{(-1)^{n+1} \Gamma(\gamma) \Gamma(\gamma+1)}{2 \Gamma(\gamma-n) \Gamma(n+\gamma+1)}+\sum_{j=1}^{2 n+1} B_{j, n, \gamma} C_{j}^{(\gamma)}(x),
$$

where the coefficients $B_{j, n, \gamma}$ are given explicitly by

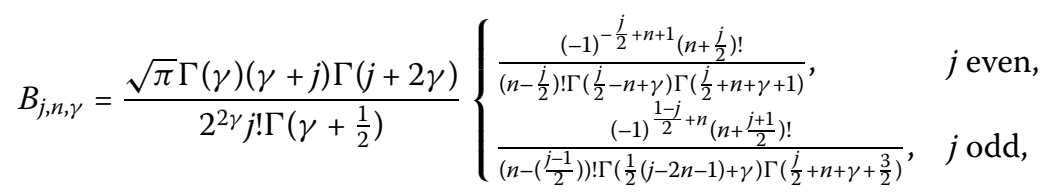

and consequently, the linearization formula (32) can be written alternatively as in (28). Corollary 6 is now proved.

In the following corollary, we state three special linearization formulas of (28).

Corollary 7 Setting $\gamma=0, \frac{1}{2}$, and 1 , respectively, in (28), yields the following three linearization formulas:

$$
\begin{aligned}
V_{n}(x) T_{n+1}(x)= & \frac{1}{2}\left(1-\xi_{n}\right)+\sum_{m=0}^{n} T_{2 m+1}(x)-\sum_{m=1}^{n} T_{2 m}(x) \\
V_{n}(x) T_{n+1}(x)= & \frac{n}{2 n+1}+\frac{\pi}{4} \sum_{m=0}^{n} \frac{(-1)^{m+n}(4 m+3)(m+n+1) !}{\Gamma\left(m-n+\frac{1}{2}\right)(n-m) ! \Gamma\left(m+n+\frac{5}{2}\right)} P_{2 m+1}(x) \\
& +\frac{\pi}{4} \sum_{m=1}^{n} \frac{(-1)^{m+n+1}(4 m+1)(m+n) !}{\Gamma\left(m-n+\frac{1}{2}\right)(n-m) ! \Gamma\left(m+n+\frac{3}{2}\right)} P_{2 m}(x), \\
V_{n}(x) T_{n+1}(x)= & \frac{1}{2}\left(2-\xi_{n}\right)+\frac{1}{2}\left[U_{2 n+1}(x)-U_{2 n}(x)\right]
\end{aligned}
$$

and $\xi_{n}$ is defined in (20). 
Now, and by following similar procedures to those followed in Corollary 6, some other linearization formulas can be obtained. Some of these formulas are stated in the following three corollaries without proofs.

Corollary 8 Setting $\alpha=\gamma=-\frac{1}{2}$, in equation (9) yields the following linearization formula:

$$
V_{n}(x) T_{n+1}(x)=\frac{1}{2}+\frac{1}{2} \sum_{j=0}^{2 n+1} \frac{\left(\begin{array}{c}
2 n+1 \\
j
\end{array}\right)(2 n+2)_{j}\left(j-2 n+\delta-\frac{1}{2}\right)_{-j+2 n+1}}{\left(j+\delta+\frac{1}{2}\right)_{j}\left(2 j+\delta+\frac{3}{2}\right)_{-j+2 n+1}} R_{j}^{\left(\frac{-1}{2}, \delta\right)}(x) .
$$

Corollary 9 Setting $\alpha=-\frac{1}{2}, \gamma=\frac{1}{2}$, in equation (9) yields the following linearization formula:

$$
\begin{aligned}
& V_{n}(x) T_{n+1}(x) \\
& =\frac{1}{2}+\frac{\Gamma\left(\delta+\frac{1}{2}\right)}{8} \sum_{j=0}^{2 n+1} \frac{(2 \delta+4 j+3)(j+2 n+1) ! \Gamma\left(j+\delta+\frac{3}{2}\right)}{j !(-j+2 n+1) ! \Gamma\left(j-2 n+\delta+\frac{1}{2}\right) \Gamma\left(j+2 n+\delta+\frac{7}{2}\right)} \\
& \quad \times(2 \delta+2 j(2 \delta+2 j+3)-8 n(2 n+3)-7) R_{j}^{\left(\frac{1}{2}, \delta\right)}(x) .
\end{aligned}
$$

Corollary 10 Setting $\alpha=-\frac{1}{2}, \delta=\gamma+1$, in equation (9) yields the following linearization formula:

$$
\begin{aligned}
& V_{n}(x) T_{n+1}(x) \\
& =\frac{1}{2}+\frac{(-1)^{n+1}\left[\Gamma\left(\gamma+\frac{3}{2}\right)\right]^{2}}{2 \Gamma\left(-n+\gamma+\frac{1}{2}\right) \Gamma\left(n+\gamma+\frac{5}{2}\right)}+\frac{\sqrt{\pi} \Gamma\left(\gamma+\frac{3}{2}\right)}{4^{\gamma+1} \Gamma(\gamma+1)} \\
& \times\left\{\sum_{m=0}^{n} \frac{(-1)^{m+n}(m+n+1) ! \Gamma(2 m+2 \gamma+3)}{(2 m+1) !(n-m) ! \Gamma\left(m-n+\gamma+\frac{3}{2}\right) \Gamma\left(m+n+\gamma+\frac{5}{2}\right)} R_{2 m+1}^{(\gamma, \gamma+1)}(x)\right. \\
& \left.+\sum_{m=1}^{n} \frac{(-1)^{m+n+1}(m+n) ! \Gamma(2 m+2 \gamma+2)}{(2 m) !(n-m) ! \Gamma\left(m-n+\gamma+\frac{1}{2}\right) \Gamma\left(m+n+\gamma+\frac{5}{2}\right)} R_{2 m}^{(\gamma, \gamma+1)}(x)\right\} .
\end{aligned}
$$

\section{Two applications for the derived formulas}

This section is concerned with presenting two applications for the linearization formulas which derived in Sections 3 and 4. In the first application, some new identities of definite integrals involving products of certain Jacobi polynomials are given. In the second application, we give some new trigonometric identities by transporting the linearization formulas to their trigonometric representations.

\subsection{Some integral formulas}

Theorem 5 Let $n$ and $m$ be nonnegative integers with $m \leq 2 n+1$. For all $\gamma, \delta>-1$, one has

$$
\begin{aligned}
& \int_{-1}^{1}(1-x)^{\gamma}(1+x)^{\delta} R_{n}^{\left(\alpha, \frac{1}{2}\right)}(x) R_{n+1}^{\left(\alpha,-\frac{1}{2}\right)}(x) R_{m}^{(\gamma, \delta)}(x) d x \\
& =\frac{\left(\begin{array}{c}
2 n+1 \\
m
\end{array}\right) 2^{\gamma+\delta+1} m !(\Gamma(\gamma+1))^{2} \Gamma(m+\delta+1)\left(\alpha+\frac{1}{2}\right)_{m}(\gamma+1)_{m}(2 n+2 \alpha+3)_{m}}{(\gamma+\delta+2 m+1) \Gamma(m+\gamma+1)(\alpha+1)_{m}(2 \alpha+1)_{m} \Gamma(m+\gamma+\delta+1)(m+\gamma+\delta+1)_{m}} \\
& \quad \times{ }_{4} F_{3}\left(\begin{array}{c}
m-2 n-1, m+\alpha+\frac{1}{2}, m+2 n+2 \alpha+3, m+\gamma+1 \\
m+\alpha+1, m+2 \alpha+1,2 m+\gamma+\delta+2
\end{array} \mid 1\right)
\end{aligned}
$$


Proof The proof follows from the linearization formulas (9) by the multiplication of its sides by $(1-x)^{\gamma}(1+x)^{\delta} R_{m}^{(\gamma, \delta)}(x)$, and then integrating over $(-1,1)$.

Some other integral formulas free of any hypergeometric functions follow from the linearization formulas obtained in Sections 3 and 4. For example, Corollaries 2 and 3 yield the following three integrals.

Corollary 11 Let $n$ and $m$ be nonnegative integers with $m \leq 2 n+1$. The following integral formulas are valid:

$$
\begin{aligned}
\int_{-1}^{1}(1-x)^{\alpha} \sqrt{1+x} R_{n}^{\left(\alpha, \frac{1}{2}\right)}(x) R_{n+1}^{\left(\alpha, \frac{1}{2}\right)}(x) R_{m}^{\left(\alpha, \frac{1}{2}\right)}(x) d x \\
=\frac{\left(\begin{array}{c}
2 n+1 \\
m
\end{array}\right) 2^{\alpha+\frac{5}{2}} m !(\Gamma(\alpha+1))^{2} \Gamma\left(m+\frac{3}{2}\right)}{(2 \alpha+4 m+3) \Gamma(m+\alpha+1) \Gamma\left(m+\alpha+\frac{3}{2}\right)} \\
\quad \times \frac{(m+2)_{-m+2 n+1}\left(\alpha+\frac{1}{2}\right)_{m}\left(m-2 n-\alpha-\frac{1}{2}\right)_{-m+2 n+1}(2 n+2 \alpha+3)_{m}}{\left(m+\alpha+\frac{3}{2}\right)_{m}(2 \alpha+1)_{m}(-2 n-2 \alpha-1)_{-m+2 n+1}\left(2 m+\alpha+\frac{5}{2}\right)_{-m+2 n+1}}
\end{aligned}
$$

and, in particular, we have

$$
\begin{aligned}
& \int_{-1}^{1} \sqrt{1-x^{2}} U_{m}(x) U_{n}(x) W_{n+1}(x) d x=\frac{\pi}{2}, \\
& \int_{-1}^{1} \sqrt{\frac{1+x}{1-x}} V_{n}(x) T_{n+1}(x) V_{m}(x) d x= \begin{cases}\frac{\pi}{2}, & m=2 n+1, \\
0, & \text { otherwise. }\end{cases}
\end{aligned}
$$

\subsection{Introducing trigonometric identities}

Some linearization formulas given in Sections 3 and 4 can be transported to give some new trigonometric identities. In the following, we give a trigonometric representation for equation (35).

Corollary 12 For all $n \geq 1$, the following trigonometric identity is valid:

$$
\begin{aligned}
& \frac{n}{2 n+1}+\frac{\pi}{4} \sum_{m=0}^{n} \frac{(-1)^{m+n}(4 m+3)(m+n+1) !}{\Gamma\left(m-n+\frac{1}{2}\right)(n-m) ! \Gamma\left(m+n+\frac{5}{2}\right)} P_{2 m+1}(\cos \theta) \\
& \quad+\frac{\pi}{4} \sum_{m=1}^{n} \frac{(-1)^{m+n+1}(4 m+1)(m+n) !}{\Gamma\left(m-n+\frac{1}{2}\right)(n-m) ! \Gamma\left(m+n+\frac{3}{2}\right)} P_{2 m}(\cos \theta) \\
& =\frac{\cos \left(\left(n+\frac{1}{2}\right) \theta\right) \cos ((n+1) \theta)}{\cos \left(\frac{\theta}{2}\right)} .
\end{aligned}
$$

Remark 4 Several trigonometric identities can be deduced from other linearization formulas obtained in Sections 3 and 4. 


\section{Acknowledgements}

The author would like to thank the anonymous referees for carefully reading the paper and for their comments, which have improved the manuscript.

Received: 31 December 2015 Accepted: 30 March 2016 Published online: 05 April 2016

\section{References}

1. Askey, R, Gasper, G: Linearization of the product of Jacobi polynomials. III. Can. J. Math. 23, 332-338 (1971)

2. Gasper, G: Linearization of the product of Jacobi polynomials. I. Can. J. Math. 22, 171-175 (1970)

3. Gasper, G: Linearization of the product of Jacobi polynomials. II. Can. J. Math. 22, 582-593 (1970)

4. Hylleraas, EA: Linearization of products of Jacobi polynomials. Math. Scand. 10, 189-200 (1962)

5. Rahman, M: A non-negative representation of the linearization coefficients of the product of Jacobi polynomials. Can. J. Math. 33(4), 915-928 (1981)

6. Chaggara, H, Koepf, W: On linearization coefficients of Jacobi polynomials. Appl. Math. Lett. 23(5), 609-614 (2010)

7. Doha, $\mathrm{EH}$ : On the construction of recurrence relations for the expansion and connection coefficients in series of Jacobi polynomials. J. Phys. A, Math. Gen. 37(3), 657-675 (2004)

8. Sánchez-Ruiz, J: Linearization and connection formulae involving squares of Gegenbauer polynomials. Appl. Math. Lett. 14(3), 261-267 (2001)

9. Doha, EH: On the connection coefficients and recurrence relations arising from expansions in series of Laguerre polynomials. J. Phys. A, Math. Gen. 36(20), 5449-5462 (2003)

10. Doha, EH, Ahmed, HM: Recurrences and explicit formulae for the expansion and connection coefficients in series of Bessel polynomials. J. Phys. A, Math. Gen. 37(33), 8045-8063 (2004)

11. Maroni, $\mathrm{P}$, da Rocha, Z: Connection coefficients between orthogonal polynomials and the canonical sequence: an approach based on symbolic computation. Numer. Algorithms 47(3), 291-314 (2008)

12. Sánchez-Ruiz, J, Dehesa, JS: Some connection and linearization problems for polynomials in and beyond the Askey scheme. J. Comput. Appl. Math. 133(1), 579-591 (2001)

13. Koornwinder, T: Positivity proofs for linearization and connection coefficients of orthogonal polynomials satisfying an addition formula. J. Lond. Math. Soc. 2(1), 101-114 (1978)

14. Foupouagnigni, M, Koepf, W, Tcheutia, DD: Connection and linearization coefficients of the Askey-Wilson polynomials. J. Symb. Comput. 53, 96-118(2013)

15. Abd-Elhameed, WM: On solving linear and nonlinear sixth-order two point boundary value problems via an elegant harmonic numbers operational matrix of derivatives. Comput. Model. Eng. Sci. 101(3), 159-185 (2014)

16. Doha, EH, Abd-Elhameed, WM: Integrals of Chebyshev polynomials of third and fourth kinds: an application to solution of boundary value problems with polynomial coefficients. J. Contemp. Math. Anal. 49(6), 296-308 (2014)

17. Doha, EH, Abd-Elhameed, WM, Bassuony, MA: New algorithms for solving high even-order differential equations using third and fourth Chebyshev-Galerkin methods. J. Comput. Phys. 236, 563-579 (2013)

18. Tcheutia, DD: On Connection, Linearization and Duplication Coefficients of Classical Orthogonal Polynomials. PhD thesis, Universität Kassel (2014). Available at https://kobra.bibliothek.uni-kassel.de/handle/urn:nbn:de:hebis:34-2014071645714

19. Doha, EH, Abd-Elhameed, WM: New linearization formulae for the products of Chebyshev polynomials of third and fourth kind. Rocky Mt. J. Math. (to appear)

20. Abd-Elhameed, WM: New formulas for the linearization coefficients of some nonsymmetric Jacobi polynomials. Adv. Differ. Equ. 2015, 168 (2015)

21. Abd-Elhameed, WM: New product and linearization formulae of Jacobi polynomials of certain parameters. Integral Transforms Spec. Funct. 26(8), 586-599 (2015)

22. Abd-Elhameed, WM, Doha, EH, Ahmed, HM: Linearization formulae for certain Jacobi polynomials. Ramanujan J. 39(1), 155-168 (2016)

23. Olver, FWJ, Lozier, DW, Boisvert, RF, Clark, CW: NIST Handbook of Mathematical Functions. Cambridge University Press, Cambridge (2010). DLMF, Digital Library of Mathematical Functions. http://dlmf.nist.gov

24. Andrews, GE, Askey, R, Roy, R: Special Functions. Cambridge University Press, Cambridge (1999)

25. Doha, EH, Abd-Elhameed, VM, Ahmed, $\mathrm{H}$ : The coefficients of differentiated expansions of double and triple Jacobi polynomials. Bull. Iran. Math. Soc. 38(3), 739-765 (2012)

26. Mason, JC, Handscomb, DC: Chebyshev Polynomials. Chapman and Hall/CRC, Boca Raton (2010)

27. Fields, JL, Wimp, J: Expansions of hypergeometric functions in hypergeometric functions. Math. Comput. 15(76), 390-395 (1961)

28. Luke, YL: The Special Functions and Their Approximations. Academic Press, New York (1969)

29. Bateman, H, Erdélyi, A, Magnus, W, Oberhettinger, F, Tricomi, FG: Higher Transcendental Functions, vol. I. McGraw-Hill, New York (1953)

30. Koepf, W: Hypergeometric Summation, 2nd edn. Springer Universitext Series. Springer, Berlin (2014). http://www.hypergeometric-summation.org

31. van Hoeij, M: Finite singularities and hypergeometric solutions of linear recurrence equations. J. Pure Appl. Algebra 139(1), 109-131 (1999) 